\title{
Una revisión de las críticas a las Teorías Marxistas de la Dependencia: Ruy Mauro Marini y los estudios sobre Chile
}

\author{
A review of the criticisms to the Marxist Theories of Dependence: \\ Ruy Mauro Marini and the studies on Chile \\ María Gabriela Rho* - Ayelén Branca**
}

\begin{abstract}
Resumen: Las Teorías Marxistas de la Dependencia (TMD) surgen en la segunda mitad de los años sesenta en el marco del debate sobre las posibilidades del desarrollo autónomo de América Latina, siendo Ruy Mauro Marini uno de sus principales representantes. Dichas teorías fueron un centro de irradiación de múltiples debates, como así también, objeto de numerosas críticas. En el presente artículo realizaremos una revisión de las críticas desarrolladas por Agustín Cueva, quien cuestionó la ausencia de articulación del análisis estructural con el de la lucha de clases en dichas teorías. Discutiremos esta tesis a través de un estudio de caso, analizando la articulación de dimensiones estructurales y súperestructurales en los escritos sobre Chile de Ruy Mauro Marini, a fin de mostrar el carácter dialéctico de estos análisis. A su vez, intentaremos exponer cómo éstos complementan sus estudios teóricos estructurales, aplicando sus conceptos en el examen de situaciones históricas concretas.
\end{abstract}

Palabras Clave: Ruy Mauro Marini; Teoría Marxista de la Dependencia; Estudios sobre Chile.

\begin{abstract}
The Marxist theories of dependence (TMD) emerged in the second half of the 1960s in the framework of the debate on the possibilities of autonomous development in Latin America, with Ruy Mauro Marini as one of its main representatives. These theories were a center of irradiation for many debates, as well as an object of numerous critiques. In this article, we will review the critiques developed by Agustín Cueva, who questioned the lack of articulation of structural analysis with that of the class struggle in these theories. We will discuss this thesis through a case study, analyzing the articulation of structural and super-structural dimensions in the writings on Chile by Ruy Mauro Marini, in order to show the dialectical character of these analyzes. In turn, we will try to show how they
\end{abstract}

\footnotetext{
* Argentina. Coautora. Profesora y Licenciada en Historia, Facultad de Filosofía y Humanidades, Universidad Nacional de Córdoba. Becaria Doctoral CONICET, CIECS (CONICET-UNC). Correo electrónico: mgabrielarho@gmail.com

** Argentina. Coautora. Profesora y estudiante avanzada de la Licenciatura en Filosofía, Facultad de Filosofía y Humanidades, Universidad Nacional de Córdoba. Correo electrónico: aye.branca@gmail.com
} 
complement their structural theoretical studies, applying their concepts in the examination of concrete historical situations.

Keywords: Ruy Mauro Marini; Marxian Dependence Theory; Studies on Chile.

Recibido: 4 mayo 2018 Aceptado: 7 julio 2018

\section{Introducción}

En la segunda mitad de los años 60, al calor de los debates sobre las posibilidades del "desarrollo" autónomo de los países latinoamericanos, surgen las Teorías Marxistas de la Dependencia (TMD) como una visión crítica respecto de las perspectivas desarrollistas sostenidas por la Comisión Económica para América Latina y el Caribe (CEPAL), así como de los posicionamientos etapistas predominantes en los Partidos Comunistas (PC) de la época. En este contexto político y académico, las TMD se presentaron como una apuesta política de intelectuales que buscaban articular su producción teórica con los procesos de luchas que se desarrollaban en el continente. De esta forma, se constituyeron como un marco interpretativo común para la intelectualidad latinoamericana (Svampa, 2016: 195), tornándose fundamental su influencia en los debates al interior de las izquierdas y del pensamiento crítico de la región.

La importancia que asumieron estas teorías las colocan como centro de irradiación de debates, como así también, objeto de numerosas críticas, que abarcan desde señalamientos teóricos hasta posicionamientos políticos ideológicos. Entre las múltiples críticas realizadas a las TMD, en el presente trabajo nos centramos en problematizar aquellas desarrolladas por Agustín Cueva, quien cuestionó la ausencia de articulación del análisis estructural con el de la lucha de clases en dichas teorías.

En miras a revaluar esta crítica, retomamos aportes teóricos y estudios de coyuntura de uno de los principales representantes de las TMD, Ruy Mauro Marini. Nuestra apuesta radica en la posibilidad de realizar un ejercicio metodológico de articulación entre los aportes teóricos y los análisis de coyuntura ya que recuperar estas dos instancias nos permitirá demostrar cómo el autor conjugó dimensiones estructurales y superestructurales al analizar la realidad social. Esto es central tanto para complejizar su desarrollo teórico, como para profundizar en sus análisis de la realidad sociopolítica, mostrando el carácter dialéctico de su pensamiento. Al mismo tiempo, nos permitirá dar cuenta de la importancia de los aportes de Marini a la construcción de un pensamiento crítico, marxista y latinoamericano en cuanto intelectual que supo conjugar su apuesta académica con su militancia política.

A los fines de los objetivos planteados, en primer lugar, realizaremos una breve introducción a las principales categorías de la TMD desarrollada por Ruy Mauro Marini en su obra La dialéctica de la dependencia (1973). Del mismo modo desarrollaremos las críticas a dicha corriente que plantea Agustín Cueva en Problemas y perspectivas de la teoría de la dependencia (1974) y la sistematización realizada por Vania Bambirra en Teoría de la Dependencia: una anticrítica (1978). En segundo lugar, abriremos un diálogo entre la teoría 
sobre la economía política de la dependencia y los análisis sobre la coyuntura chilena de la década del 70 desarrollados por Marini. Así, recuperaremos sus trabajos de análisis y reflexión sobre la realidad sociopolítica chilena reunidos en el libro El Reformismo y la Contrarrevolución. Estudios sobre Chile (1976) ${ }^{1}$. Al aproximarnos a esta obra, proponemos una división basada en una decisión analítica y metodológica, según la cual, identificamos dos momentos de análisis, que podríamos denominar estructural y superestructural. Cabe destacar que, si bien Marini no distingue dichos momentos en el desarrollo de su obra, podemos identificar que estas dimensiones aparecen con mayor y menor preponderancia a lo largo de sus estudios. Encontramos que la distinción no es arbitraria, sino que responde a categorías de análisis que se desprenden de la teoría marxista. Dichas categorías suscitaron múltiples debates en torno a su definición, articulación y condicionamiento mutuo.

Recuperando a Marx (1859) entendemos por estructura económica de la sociedad a las relaciones de producción que se corresponden a una determinada fase de desarrollo de las fuerzas productivas materiales. Sobre esta base material, afirma Marx (1859), se "levanta la superestructura jurídica y política y a las que corresponden determinadas formas de conciencia social". Es decir, entendemos por superestructura las formas políticas y jurídicas que asume la lucha de clases y su resultado; las formas en que estas se cristalizan en la conciencia de clase y la vida política, cultural, intelectual y espiritual de la sociedad. Si bien Marx habla de la estructura como la "base material" de la superestructura, cabe destacar, recuperando a Dos Santos (2011: 29), referente de las TMD, que existe una relación dialéctica de determinación (de precedencia lógica, causal, histórica y material) de la base material sobre la superestructura institucional, política y cultural, lo que no significa que éstas sean total o fundamentalmente pasivas. Interpretación que se desprende de las mismas lecturas de otros estudios marxistas previos como los de Engels que afirma que "la situación económica es la base, pero los diversos factores de la superestructura que sobre ella se levanta (...) ejercen también su influencia sobre el curso de las luchas históricas y determinan, predominantemente en muchos casos, su forma" (Engels, 1980).

En los estudios de Marini, como parte de la tradición marxista, podemos identificar elementos estructurales y superestructurales en distintos momentos de su análisis y, en sintonía con lo planteado por Engels y Dos Santos, dicha división no se presenta de manera categórica ni taxativa, sino que, en los diferentes estudios del autor, encontramos articuladas ambas dimensiones de análisis. Cabe destacar que hay estudios teóricos como la Dialéctica de la dependencia en el que prima un análisis estructural y es aquí donde se centran las críticas a Marini.

\footnotetext{
${ }^{1}$ En palabras del autor el propósito del libro es ofrecer a los lectores un conjunto de textos, publicados de maneras dispersas, que tienen como objetivo reflexionar sobre la estrategia y práctica revolucionaria en el proceso chileno de 1970-1973. La mayoría de los textos reunidos en este libro fueron escritos durante este periodo y publicados en prensa de la época como Chile Hoy, a excepción del primer apartado, "Dos estrategias en el proceso chileno", publicado un año después; y, de la parte cuatro del libro "Economía política de un golpe militar", redactado dos meses después del golpe militar y posteriormente reelaborado (Marini, 1976: 9).
} 


\section{Notas sobre la teoría marxista de la dependencia en el pensamiento de Ruy Mauro Marini}

Ruy Mauro Marini fue uno de los principales referentes de las TMD, destacándose por su rigor metodológico, como así también, por sus numerosos artículos periodísticos y de coyuntura que analizan de forma crítica la realidad latinoamericana de su época. En tanto militante que supo conjugar su actividad académica con una activa participación política, se vio obligado a vivir entre la persecución y el exilio durante las décadas del 60 y 70 . El golpe de Estado en Brasil de 1964 lo obligaron a exiliarse a México, lugar del que también se vio forzado a dejar por el gobierno en el año 1969. En este momento se dirigió a Chile, país que abandona en el año 1973 a causa de la caída de Allende y el inicio de la dictadura de Pinochet. Este escenario de exilio, marcado por la persecución política, caracteriza el desarrollo académico e intelectual de la época en la cual se inscribe las teorías de la dependencia ${ }^{2}$ y, en particular, su versión marxista. De este modo, la dimensión teórica es inseparable de la militancia política de sus principales referentes, siendo Brasil, Chile y México los centros de producción de las TMD ${ }^{3}$.

Por su parte, la Revolución Cubana fue clave en el desarrollo del pensamiento marxista latinoamericano, destacándose como como momento de quiebre en la tradición de izquierda y revolucionaria del continente ${ }^{4}$. La experiencia cubana se había convertido en una referencia obligatoria para los revolucionarios latinoamericanos, quienes no sólo la recuperan para los análisis económicos y sociales, sino como hito para pensar estrategias políticas en el continente (Briceño Ramírez, 2016: 94; Lozoya, 2016: 103). Este proceso se constituyó, entonces, como una referencia a partir de la cual se incorporan elementos políticos para pensar y estudiar las distintas realidades locales. Si bien, hay perspectivas en los que se convirtió en un espejo y una receta a seguir, también surgen posiciones críticas, como las de la nueva militancia revolucionaria chilena, en las que se incorporan elementos sin caer en una aplicación estática del proceso.

Los primeros intentos de elaboración de las tesis sobre la dependencia surgieron en Brasil a principios de la década del 60, de la mano de intelectuales y docentes de la Universidad de Brasilia como André Gunder Frank, Victor Nunes Leal, Theotonio Dos Santos, Vania Bambirra y Ruy Mauro Marini; muchos de los cuales fueron a la vez fundadores y referentes de Política Operaria, organización de izquierda revolucionaria que buscó disputar las tesis sostenidas por los PC de la época (Martins, 2015: 9-10). Fruto de los debates impulsados por los militantes e intelectuales de esta organización, es que surge una corriente específicamente marxista, en la cual se circunscribe el desarrollo de Marini, especialmente en los estudios realizados en el periodo de 1973 a 1979.

Fue en Chile donde estas tesis pudieron ser sistematizadas y elaboradas de manera más definitiva, ya que el fecundo ambiente político, académico y de discusión que se instala, da las

\footnotetext{
${ }^{2}$ Lo que posteriormente se denominan "teorías de la dependencia" deben ser enunciadas en plural ya que en su interior se reúnen diversas de perspectivas y líneas de investigación, tal como lo demuestran los estudios de Amaral (2006), Astarita (2010), Beigel (2006), Dos Santos (2002), Katz (2016), entre otros.

3 Marini participó en la redacción de notas periodísticas en diarios de los diferentes países en los que vivió a lo largo de su vida. Por ejemplo, en Brasil, en el año 1964, escribió para O Metropolitano y entre los años 1972-1973, en Chile Hoy. En México publicó en Excélsior (1975-1976), en Sol de México (1976-1977) y en El Universal (1977-1979).

4 Para comprender la repercusión de la Revolución Cubana en el desarrollo del pensamiento social de América Latina, consular: Sigal (1991: 149- 205), Terán (1993: 175-205), Bambirra (1976), Löwy (2007) y Astarita (2010).
} 
condiciones para que las TMD se desarrollen (Bambirra, 1978: 21). Este contexto estuvo marcado tanto por la disputa electoral de 1970, que abrió grandes debates al interior de la izquierda y el movimiento popular, como por el hecho de que en Chile se encontraban las sedes de la CEPAL y del Instituto Latinoamericano de Planificación Económica y Social (ILPES). En estas instituciones trabajaron Henrique Cardoso, Aníbal Quijano, Francisco Welffort, entre otros, quienes hicieron grandes contribuciones a los estudios de la dependencia y con los cuales, los intelectuales dependentistas de tradición marxista, nucleados en equipos de investigación del Centro de Estudios Socio Económicos (CESO), entablaron profundos debates (Bambirra, 1986: 22-23).

En sintonía con el clima político chileno, Marini comenzó a militar en el Movimiento de Izquierda Revolucionaria (MIR), a formar parte del CESO y a dictar seminarios y cursos sobre teoría del cambio, el problema de la transición al socialismo, teoría marxista y realidad latinoamericana (Martins, 2015: 11). Es en su segundo exilio donde resultaron los dos trabajos analizados en este artículo, Dialéctica de la dependencia (1973) -obra que inauguró lo que podría denominarse la "economía política de la dependencia"- y los artículos reunidos en El reformismo y la contrarrevolución. Estudios sobre Chile (1976), en los cuales realizó un análisis y balance de la experiencia chilena.

El punto de partida teórico de Marini se encuentra en la teoría general del modo de producción capitalista presentada específicamente en El Capital, así como en su interés por analizar las particularidades que éste asume en América Latina. Su esfuerzo radicó en poder comprender la realidad latinoamericana, corriéndose de aquellos estudios marxistas que caen en perspectivas eclécticas negando el mismo marxismo; o bien, aquellas perspectivas que, queriendo aplicar el modelo sin más, se sesgan en miradas abstractas e inútiles (Marini, 1973: 107-108). En este sentido, la lectura del autor sobre el proceso chileno, sus críticas a las políticas económicas desarrollistas de la Unidad Popular (UP) y a las estrategias de alianza y análisis políticos del PC, se comprenden en el marco general de las condiciones de la región determinado por las características propias del capitalismo dependiente. Ahora bien, qué entiende Marini por capitalismo dependiente, qué particularidades asume el mismo en la coyuntura chilena analizada.

En el marco de una disputa directa con la perspectiva desarrollista que se expandía en la región y, al mismo tiempo, con el etapismo asumido por la posición hegemónica del PC, Marini intentó comprender la realidad latinoamericana como parte de la expansión global del capitalismo. Por un lado, buscaba superar el dualismo estructural presente en los análisis cepalinos $^{5}$. A diferencia de esta perspectiva que divide espacial y temporalmente al sistema económico y político mundial entre centros y periferias como dos estructuras diferenciadas que no se condicionan mutuamente, Marini entiende dichos antagonismos como dos expresiones dentro de un mismo proceso histórico de expansión del capitalismo. El desarrollo de unas partes del sistema se produce a expensas del subdesarrollo de otras, siendo la dependencia tanto consecuencia como condición de posibilidad de dicho proceso. Por otro lado, en contra de la caracterización del momento histórico sostenida por el PC, según la cual en América Latina aún no se ha desarrollado el modo de producción capitalista ni se ha llevado adelante revoluciones democrático burguesas, Marini caracteriza la etapa histórica de la región como

5 Sobre los aportes de la CEPAL consultar: Cardoso (1977), CEPAL (1951), Furtado (1982), Presbich (1986) y Rodrigez (1980). 
capitalismo dependiente ${ }^{6}$. Es decir, sostiene que América Latina es capitalista, pero en la región el capitalismo se manifiesta como dependiente.

Desde una perspectiva dialéctica y sin pretensiones de postular una teoría general, a partir del análisis de los mecanismos característicos de determinado modo de producción (y circulación), que asume diferentes complejizaciones y particularidades en las distintas fases del desarrollo capitalista, Marini desarrolló la categoría de dependencia como una "relación de subordinación entre naciones formalmente independientes, en cuyo marco las relaciones de producción de las naciones subordinadas son modificadas o recreadas para asegurar la reproducción ampliada de la dependencia" (Marini, 1973a: 111).

Comprender en profundidad el concepto de dependencia obliga a volver a la teoría de valor de Marx, específicamente la configuración de la ley del valor en el ámbito de la circulación de capitales desarrollada en el tomo III de El Capital (2009b). Aquí se presenta la relación de dependencia como uno de los mecanismos que contrarresta la caída tendencial de la tasa de ganancia en el capitalismo central. Retomando esta idea Marini explicó el intercambio desigual y la transferencia de valor a los centros capitalistas, desde una perspectiva que complejizó la concepción cepalina de la desigualdad en el intercambio basada en el tipo de bienes producidos, ya que integró en el análisis de la transferencia de valor, la fijación de los precios de mercado y los precios de producción de mercancías. Del mismo modo, muestra que la transferencia de valor tiene como consecuencia la interrupción del ciclo de valorización del capital a escala local: parte del valor producido en América Latina no puede ser acumulado en la economía local. Esta interrupción es saldada por los capitalistas de países dependientes en el plano de la producción:

Lo que aparece claramente, pues, es que las naciones desfavorecidas por el intercambio desigual no buscan tanto corregir el desequilibrio entre los precios y el valor de sus mercancías exportadas (lo que implicaría un esfuerzo redoblado para aumentar la capacidad productiva del trabajo), sino más bien compensar la pérdida de ingresos generados por el comercio internacional, recurriendo a una mayor explotación del trabajador (Marini, 1973a: 123).

La "mayor explotación del trabajador" es lo que Marini denominó superexplotación, que puede definirse como el pago de la fuerza de trabajo por debajo de su valor, el cual está representado por el costo de reproducción de la fuerza de trabajo y constituye una característica estructural del capitalismo dependiente (Marini, 1973a: 123-124). Hay tres mecanismos que la definen, a saber, el aumento de la intensidad del trabajo, la prolongación de

${ }^{6}$ Tanto la discusión con el PC como con la perspectiva cepalina están estrechamente vinculadas con debates políticos. Por un lado, el debate con el PC se centra en su política de alianza de clases. Este planteaba que la revolución en lo inmediato no tenía que ser socialista, sino que democrático burguesa, agraria y anti-imperialista, por lo que, consideraban necesario aliarse con todos los sectores que aportaron a cumplir las tareas de la revolución democrático burguesa y, de ahí que, la burguesía nacional se entendía como un aliado central en este primer momento de la revolución. Esta postura del PC chileno es duramente criticada por Marini, considerando que a partir de esta política aliancista del PC se generaron dos líneas en la izquierda que provocaron una falta de unificación y debilidad de la UP en la dirección del movimiento de masas. Por otra parte, y con respecto a la CEPAL, Marini critica la política desarrollista que se expresa en el optimismo por el proceso de sustitución de importaciones asumiendo que, mediante la política de gobiernos, es posible superar la condición de dependencia. Ante lo cual Marini planteaba que la relación de dependencia no se supera con el proceso de sustitución de importaciones, sino que se complejiza, puesto que los países dependientes además de bienes de consumo industrializados, requieren del exterior bienes de capital para desarrollar la industria interna. A diferencia de la perspectiva desarrollista, el proceso sustitutivo no garantiza una vía al desarrollo, sino que supone un vínculo más complejo de dependencia. 
la jornada laboral y la reducción del consumo del obrero (Marini, 1973a: 124-125). Este último mecanismo es el que específicamente se desarrolla en América Latina ya que, al destinar su producción a las necesidades del mercado mundial, el consumo no depende, de la capacidad interna. Se daría así una escisión entre las esferas de circulación y producción. A diferencia de lo que sucede en el "capitalismo clásico", los trabajadores pierden su doble carácter de productores y consumidores y se ven reducidos a meros productores. Marini planteó, entonces, una mirada particular acerca de cómo se constituyen y definen las clases sociales fundamentales en el capitalismo dependiente, entendiéndolo como un proceso diferente (aunque relacionado) con las características de las mismas clases en los países imperialistas centrales (Marini, 1973a: 125).

El concepto de superexplotación fue uno de los aportes centrales de Marini a las TMD, convirtiéndose también en uno de los principales puntos de debate y cuestionamiento. Pues, en término generales, las críticas se centran en señalar que, desde la perspectiva de las TMD, el capitalismo en la región se reproduce por una mera imposición coercitiva de la explotación a la clase trabajadora, sin tener en cuenta cómo la lucha de clases atraviesa constitutivamente todas las esferas del capital, sea en la producción o en la reproducción (Cueva, 1974: 80-81). Al mismo tiempo que, lecturas centradas en La Dialéctica de la Dependencia, incurren en la idea de que la constitución de las clases sociales de América Latina queda determinada únicamente en función de las necesidades del mercado internacional. Mientras el desarrollo de la burguesía local se encontraría limitada por el mecanismo de la transferencia de valor, la clase trabajadora se define y constituye estrictamente por la superexplotación. En este sentido, críticas como las que realiza Agustín Cueva encuentran en esta categoría los fundamentos para señalar que en los estudios de Marini no hay una articulación entre el análisis estructural con el de la lucha de clases:

Es el análisis de las clases y su lucha lo que constituye el talón de Aquiles de la teoría de la dependencia. Para empezar, los grandes y casi únicos protagonistas de la historia que esa teoría presenta son las oligarquías y burguesías, o, en el mejor de los casos, las capas medias; cuando los sectores populares aparecen, es siempre como una masa amorfa y manipulada por algún caudillo o movimiento populista; de suerte que uno se pregunta por qué en Brasil, por ejemplo, se estableció un régimen claramente anticomunista (y no antipopulista), o cómo fue posible que en Chile se constituya "de repente" un gobierno como el de la Unidad Popular (Cueva, 1974: 74).

En La dialéctica de la dependencia Marini no profundiza en las condiciones sociales y políticas que explican la pertinencia de la categoría de superexplotación y el modo en que caracteriza las clases sociales $y$, particularmente, a los trabajadores, pareciera que remite solo a condicionantes estructurales. Esta lectura sugiere que el lugar de la clase trabajadora en la lucha de clases está totalmente determinado por estos condicionantes. Ante esta interpretación es importante recuperar los señalamientos del propio autor respecto a la metodología y objetivos de la investigación. Marini plantea que no está analizando la totalidad de la realidad social latinoamericana sino las condiciones estructurales en las que se inscribe la lucha de clases (Marini, 1973a: 149). En este sentido, sostiene que la dimensión estructural es solo un momento del análisis y reconoce que "las implicaciones de la superexplotación trascienden el plano del análisis económico y deben ser estudiadas también desde el punto de vista sociológico y político" (Marini, 1973b: 163-164). El análisis de las condiciones estructurales es, en el 
materialismo, un momento clave para el estudio de la realidad social, lo que no implica que esta dimensión determine de forma mecánica el resto de los aspectos constitutivos de aquella. En línea con el desarrollo de Marini, Vania Bambirra (1978) responde a estas críticas planteando que:

El análisis de clases es, en primer lugar, un análisis de la estructura económica -para ser más inteligibles y rigurosos (a Agustín Cueva le gusta el rigor): un análisis de la estructura y desarrollo de las fuerzas productivas y de las relaciones de producción. Luego, un análisis de su movimiento, vale decir, de la lucha de clases, y, en seguida, un análisis de sus manifestaciones superestructurales, esto es las ideologías, instituciones, cultura, etcétera. Analizar la dependencia solamente al nivel de las manifestaciones tangibles de la lucha de clases, o prescindir de ésta y limitarse al nivel puramente del sistema productivo, sólo se justifica como momentos de la investigación que necesitan enseguida ser integrados en una perspectiva más amplia y totalizadora. Por lo general esta perspectiva totalizadora fue lograda en múltiples trabajos sobre la dependencia, aunque es obvio que los diversos autores hayan privilegiado en algunas investigaciones parciales algunos aspectos del fenómeno. De ahí proviene el riesgo de ligereza analítica si se consideran partes y no el conjunto de la obra de un autor (Bambirra, 1978: 44).

De este modo, observamos que las críticas señaladas a Marini, se sostienen sobre una visión sesgada de la metodología vinculada al materialismo dialéctico, así como de la obra del autor. Si bien en La dialéctica de la dependencia hay una preponderancia de análisis estructurales y abstractos que se dirigen hacia lo concreto, en otros de sus trabajos, se ve con claridad la articulación de las diversas dimensiones, estructurales y superestructurales, que dan cuenta de la complejidad de la realidad social y de la totalidad en los análisis que surgen tras una teoría que se concibe heredera de la tradición marxista. En este sentido cabe destacar obras previas como La dialéctica del desarrollo capitalista de Brasil, de 1966, y posteriores, como el El reformismo y la contrarrevolución. Estudios sobre Chile, escrita en el 1976, analizada a continuación.

\section{Estudios sobre Chile: las condiciones estructurales del capitalismo dependiente chileno en el periodo de ascenso de la Unidad Popular}

En la explicación de fenómenos sociopolíticos Marini, inscripto en la tradición marxista, sostiene que es necesario hacer, en un primer momento, un análisis de la estructura económica y del desarrollo de las fuerzas productivas del periodo histórico que se busca analizar. Así, sus interpretaciones sobre la realidad social y, las disputas con otras lecturas y posicionamientos políticos, se vincularon directamente a un estudio riguroso sobre las condiciones materiales de determinada coyuntura. Esto se refleja con claridad en los estudios sobre Chile, en los cuales sostuvo que la victoria de la UP no puede explicarse únicamente a partir de las estrategias y alianzas políticas desplegadas por los diversos partidos ya que consideraba que la "política no se decide exclusivamente, y ni siquiera primariamente, en el plano de lo político" (Marini, 1976: 56). De este modo, rechazó las lecturas que afirman que el ascenso de la UP se debe a un error de cálculos de la burguesía considerando que, para entender el escenario de contienda electoral de 1970, es necesario partir de un análisis de las bases objetivas que llevaron a la burguesía a 
presentarse dividida en dichos comicios ${ }^{7}$. Las candidaturas de Alessandri (Partido Nacional) y Tomic (Democracia Cristiana) mostraban para el autor la crisis del sistema de dominación burgués que operaba en Chile desde fines de los años 30 y consideraba que, para entender estas divergencias, se deben buscar explicaciones en los cambios estructurales operados en el aparato productivo chileno. En este sentido, para Marini las contradicciones interburguesas que se reflejan en el plano político pueden ser explicadas en mayor medida por el "proceso mismo de acumulación del capital o del desarrollo capitalista que tuvo lugar en la industria chilena, en el curso de la década pasada" (Marini, 1976: 57).

El autor planteaba que el conflicto económico y social desatado a fines de la década del 60 se debió a que, durante el gobierno de Frei Montalva (1963-1970), se produjo una caída del ritmo de crecimiento y una desigualdad en la evolución de los subsectores y ramas de la industria. En este proceso, se produjo un desplazamiento del eje de acumulación de capital desde las industrias tradicionales de bienes de consumo y bienes intermedios que producían para ellas -donde predominaban la mediana y la pequeña burguesía- hacia las llamadas industrias dinámicas, dedicadas a la producción de bienes más sofisticado y suntuarios -en donde el predominio cabía al gran capital nacional y extranjero (Marini, 1976: 14). Así, se produjo un estancamiento en la producción de aquellos bienes dirigidos al consumo interno, principalmente, de las clases de bajos ingresos y, al mismo tiempo, se registró una expansión de la producción de bienes suntuarios destinados al consumo de las clases con gran poder adquisitivo.

De forma paralela a este proceso, se produjo una tendencia a la monopolización en todas las ramas de la industria chilena, acentuada principalmente, en las ramas de alta tecnología (Marini, 1976: 70). La monopolización venía desarrollándose en Chile desde la década del 50, donde la expansión económica daba lugar a la concentración de capitales en base a su reproducción ampliada ${ }^{8}$. Si bien este proceso generó desequilibrios entre grandes, medianas y pequeñas empresas, mientras perduró el dinamismo económico, los vínculos permanecieron armónicos. Sin embargo, con la contracción económica de los 60, se empezó a acentuar las divergencias, al mismo tiempo comenzaron a desarrollarse procesos de centralización del capital, donde capitales mayores crecieron a partir de la absorción de capitales menores. Esta situación, sumada al mayor desarrollo de las empresas receptoras de capital y tecnología extranjera9 ${ }^{9}$ conllevó a la intensificación de las contradicciones interburguesas y así, al deterioro entre sus relaciones políticas.

7 Marini sostiene que la victoria de Salvador Allende no puede ser explicada por la cantidad de votos obtenidos por la UP en las elecciones de 1970 ya que, prácticamente, mantiene el mismo porcentaje que en elecciones anteriores. De este modo, para el autor, la explicación debe buscarse en los motivos que llevaron a los partidos opositores de la burguesía a no unirse en dicha contienda electoral y tomar la decisión de presentarse por separado (Marini, 1976: 12).

8 Cabe diferenciar el proceso de concentración de capital del de centralización: "la concentración corresponde a un proceso de monopolización caracterizado por la expansión de un capital dado, con base en su propia reproducción ampliada, y se distingue de otro proceso de monopolización, el de la centralización en el cual un determinado capital absorbe otros capitales ya formados" (Marini, 1976: 70).

${ }^{9}$ Marini señaló que el capital extranjero incide básicamente en la esfera de la gran producción: "la participación extranjera significa, por lo general, un nivel tecnológico más alto en la empresa en que se da y corresponde a un menor gasto en sueldos y salarios, lo cual, además de evidenciar la depresión del empleo, puede indicar también que la menor ocupación que se da en la empresa tecnológicamente más avanzada se acompaña del mantenimiento del nivel promedio de remuneración en el sector, conllevando así a la formación de una plusvalía extraordinaria" (Marini, 1976: 78). 
Las transformaciones en la base productiva de la economía chilena están vinculadas a las características que asume el capitalismo dependiente, a la vez que refleja el modo en que se reproduce la dependencia. Las TMD buscan comprender el carácter y las contradicciones del capitalismo dependiente en su fase de integración monopólica mundial dando cuenta que la imposibilidad del desarrollo nacional dependiente está condicionada por el imperialismo, como un fenómeno constitutivo de estas naciones. En el caso chileno puede verse con claridad cómo el proceso de industrialización y monopolización no lleva a un proceso de independencia económica, sino a la profundización de las características de economías dependientes. Esto se explica por las condiciones sociales en las que se estructura la dependencia, donde, la acumulación del capital del sector dominante de la burguesía, no depende del desarrollo de las industrias de bienes corrientes ni del desarrollo del consumo interno ya que, como señalamos anteriormente, garantiza sus ganancias por la superexplotación de la clase trabajadora. El consumo interno de bienes corrientes no es una preocupación ni una prioridad para los capitalistas de la región, en la medida en que, en el capitalismo dependiente la clase trabajadora pierde, en relación a los países centrales, su doble condición de fuerza de trabajadora y consumidora.

Para continuar con el estudio de los factores que explican el ascenso al gobierno de la UP -luego de analizar los cambios estructurales en la economía chilena- Marini pasa a un segundo momento de análisis. Así, muestra las transformaciones que se producen en la estructura de clases chilenas a partir del desplazamiento en el sistema de acumulación y la monopolización de la industria. Entre las principales transformaciones se encuentran: el surgimiento de contradicciones interburguesas; el ascenso del movimiento de masas en el campo y la ciudad; la permanencia de la pequeña burguesía propietaria como clase significativa y el surgimiento de nuevas capas pequeñoburguesas no propietarias.

\section{Crisis del sistema de dominación: las contradicciones interburguesas}

Las transformaciones en el sistema productivo chileno generaron una agudización de las contradicciones interburguesas que provocaron una profunda crisis en el sistema de dominación burgués. Contradicciones que, a su vez, fueron reforzadas por las políticas económicas del gobierno de Frei Montalva, quien, desde 1967, tomó medidas orientadas en beneficio del desarrollo del gran capital. Por un lado, benefició a las grandes industrias, mediante el financiamiento público, la otorgación de créditos y el desarrollo de infraestructura y, por otro lado, adoptó una política regresiva de distribución de ingresos, "capaz de promover una adecuación de las estructuras de consumo en favor de la producción suntuaria" (Marini, 1976: 15).

Si bien la principal ruptura de intereses entre las capas burguesas se dio en el plano industrial, Marini señalaba que dichas contradicciones también comenzaron a manifestarse en el campo como consecuencia del impulso de la reforma agraria y de las políticas de sindicalización rural a gran escala. La reforma agraria, en un plano externo, seguía los lineamientos de la Alianza para el Progreso elaborada por Estados Unidos para la región latinoamericana y, en el plano local, buscaba atender las presiones que se daban entre algunos sectores de la DC para contener el agravamiento de la lucha de clases, la radicalización del movimiento campesino y contrarrestar la propaganda política de la izquierda chilena. A la vez que la DC entendía las políticas de sindicalización rural como una estrategia política que 
apuntaba a ampliar la base de apoyo para su partido. El impulso de estas medidas por el gobierno de Frei Montalva inevitablemente generó un nivel de conflicto y enfrentamiento con la clase terrateniente, es decir, con los grandes latifundistas, sectores claves en el sistema de dominación chileno.

En este punto observamos, cómo Marini articula variables estructurales y superesturales ya que muestra que en parte, las transformaciones del modo de acumulacion, fueron condicionadas por las políticas tomadas por el gobierno de la DC. En este sentido, la disputa política entre la DC y los sectores de izquierda, producto de las luchas de clases, juegan un rol fundamental para explicar el comportamiento político de la clase terrateniente chilena. En contraposición a las críticas de Cueva según la cual la lucha de clases y la organización de los sectores populares quedan excluidos en los estudios del autor, aquí se visualiza la incorporación de estos elementos en el análisis.

La crisis en el sistema de dominación tanto en el campo como en la ciudad, se hizo visible y se profundizó en 1970 cuando la pequeña y mediana burguesía -perjudicadas por la política del gran capital que imponía el gobierno de Frei Montalva- no logró nuclear en torno a Tomic el apoyo de la gran burguesía y de los sectores más conservadores de las capas medias y pequeño burguesas, pese al intento de repetir las alianzas establecidas en 1964. Es así como, para Marini, la fragmentación y divergencia de la burguesía en el plano económico como político, que condujeron a la crisis del sistema de dominación, se transformaron en uno de los factores centrales para explicar las particularidades de las elecciones de 1970 que dan como resultado la victoria y el ascenso al gobierno de la UP.

\section{El lugar de la pequeña burguesía en la economía chilena y su rol político}

En los países periféricos la pequeña burguesía propietaria -a diferencia de lo que sucede en los países centrales donde ésta tiende a desaparecer con la expansión capitalista- mantiene una posición significativa, debido a las particularidades del capitalismo dependiente. Esta pervivencia de la pequeña burguesía radica tanto en las dificultades de acumulación de capital en la región como, en la imposibilidad de la competencia comercial en el mercado interno en el marco del proceso de monopolización. A su vez, tal como destacó Marini, otra característica del capitalismo dependiente es la expansión de los servicios públicos y privados, la cual trae como resultado el surgimiento de nuevas capas pequeñoburguesas no propietaria, es decir, las modernas clases medias. Esta clase se origina de la proletarización de la pequeña burguesía propietaria y, en este sentido, conserva sus hábitos, su ideología y reacciones frente al proletariado y la burguesía. Por lo que, si bien es una clase asalariada y, por lo tanto proletarizada, en un plano político, ideológico y cultural se entiende como parte de la burguesía. En este momento del análisis es interesante el modo en que Marini articula elementos estructurales (posición en las relaciones de producción) y superestructurales (hábitos e ideologías) a la hora de caracterizar a la pequeña burguesía, lo que da cuenta que para el autor las clases no solo se definen por la posición que ocupan en la estructura productiva sino también por su posicionamiento político e ideológico.

Las características de la pequeña burguesía hacen que tenga una posición significativa en la estructura social y en la disputa política chilena. A diferencia del resto de América Latina ${ }^{10}$,

10 Marini afirma: "a diferencia de México, en donde esta capa utiliza la posición en el Estado para ascender a las filas de la burguesía, dando origen a lo que se ha llamado burguesía democrática, o de Brasil, en donde, además de su 
en Chile la pequeña burguesía, se logró posicionar como una fuerza política autónoma constituyéndose como árbitro del conflicto que opone a la burguesía y al proletariado. Como una verdadera capa política dirigente, que responde a los intereses de su clase, se aliaba, en determinados contextos, con las capas burguesas o apoyaba los proyectos políticos de los sectores proletarios en los momentos en que no se encontraba amenazada. Esta situación se reflejó en su comportamiento contradictorio y fluctuante a partir de 1964: en 1970 se separó de la gran burguesía, presentando un candidato propio (Tomic); en los primeros años del gobierno UP se convirtió en un actor político neutral, cuando las políticas económicas la beneficiaban; y, con la crisis de 1972, comenzó a jugar un papel relevante a favor de la gran burguesía que termina con el golpe de estado de 1973. De este modo, tanto la UP como los sectores más conservadores vinculados al gran capital, la privilegiaron como aliada fundamental, lo que acentuó a su vez la autonomización relativa que tuvo en la última década y su poder como árbitro en la disputa política chilena (Marini, 1976: 113-115).

\section{El ascenso del movimiento de masas en el campo y la ciudad}

Marini identificó en Chile un marcado ascenso del movimiento de masas urbanas y campesinas. La prioridad que le dio en estos estudios a este momento de análisis, muestra la importancia para el autor del movimiento de masas para explicar el ascenso de la UP. En efecto, en los estudios sobre Chile, encontramos un apéndice dedicado específicamente al estudios de dicha variable para la comprensión del período (Marini, 1976: 168-204), lo que permite problematizar una de las críticas realizadas por Agustín Cueva11.

En palabras de Marini, "la victoria de la UP fue a la vez el producto de un formidable proceso de movilización y radicalización del movimiento de masas y una señal verde, un incentivo, para la profundización y el ensanchamiento del movimiento de masas" (Marini 1976: 168). En esta línea, el autor divide el estudio de la dinámica del movimiento de masas en dos períodos, uno que va del 1967 a 1970, que comprende los últimos años del gobierno de Frei, y otro del 1971 al 1972 durante el gobierno de Allende.

En el primer periodo el autor identificó un aumento de las huelgas desde comienzos de los años 60 como así también, un aumento en los procesos de sindicalización de los trabajadores. No fueron los sectores de punta, es decir, los trabajadores industriales los que participaron más activamente de estas movilizaciones, sino que fueron los sectores no tradicionales -campesinos y obreros de la construcción- los protagonistas de este proceso de ascenso de lucha. Este ascenso responde tanto a la crisis económica abierta por el gobierno de la DC y su incapacidad de dar respuesta, como a elementos políticos e ideológicos presentes en dicho gobierno. Tal como señalamos anteriormente, el despertar del movimiento campesino se

subordinación efectiva a la burguesía, sufre una degradación progresiva, pasando a vegetar en los puestos subalternos del aparato estatal, la pequeña burguesía burocrática en Chile logra mantener la posición conquistada sin que esto acarree para ella el desprendimiento de su clase y su incorporación a la burguesía" (Marini, 1976: 105106).

11 Marini señala en la Presentación de los estudios sobre Chile haber realizado un examen de la evolución del movimiento de masas, pero el mismo se perdió en manos de los militares que quemaron dichos escritos, entre otros. Por lo que, en la recopilación final el autor analiza la evolución del movimiento huelguístico y los procesos de sindicalización de los trabajadores rurales y urbanos solo con "datos provenientes del servicio de información del Movimiento de Izquierda Revolucionaria; esos datos, basados en fuentes oficiales, extraoficiales y del propio MIR, se publican como anexos a informes y resoluciones de su Comité Central." (Marini, 1976: 9). 
generó a partir del proceso de sindicalización rural impulsado por la DC como estrategia política para reconstruir y ampliar las bases de apoyo de su partido; paralelamente, los trabajadores excluidos beneficiados por la reforma, iniciaron un proceso de lucha bajo formas poco ortodoxas como, por ejemplo, las tomas de tierra, corridas de cerco y el aumento de huelgas ilegales.

La importancia del movimiento de masas para la victoria de la UP se reflejó en el hecho de que es en el año 1970 cuando se da la mayor movilización. Al mismo tiempo, el gobierno habilitó condiciones de lucha que fomentaron la organización y movilización de las masas. En el año 1971, se sumaron a los sectores campesinos y de la construcción, trabajadores del sector minero, empleados fiscales y de servicios y trabajadores de la pequeña y mediana industria y comercio. Cabe destacar que para el autor el estudio y análisis aislado del movimiento de masas de por sí no es explicativo, debido a que es necesario considerar el conjunto de la correlación de fuerzas entre las clases.

\section{Estudios sobre Chile: debates políticos y lucha de clases}

En El Reformismo y la contrarrevolución, Marini introdujo los factores que habilitaron el golpe de Estado al gobierno de Allende por parte de los sectores militares y de la derecha chilena. En la presentación de estos factores complejizó su estudio integrando dimensiones sociales y políticas en su análisis. El estudio de Marini amplía el análisis estructural con el desarrollo de la dinámica del desenlace de la lucha de clases y sus manifestaciones superestructurales. En las diferencias tácticas y estratégicas de la izquierda y, en la contraofensiva burguesa, se encuentra las condiciones que dieron lugar y allanaron el terreno para el golpe de estado en 1970.

\section{Las líneas al interior de la izquierda}

El debate al interior de la izquierda chilena se encuentra atravesado por las lecciones político estratégicas de la Revolución Cubana que "nutrieron el pensamiento marxista latinoamericano, aportando a la conformación de una cultura política revolucionaria, la cual desarrolló una teoría y praxis en que la lucha por el socialismo era una lucha política e insurreccionalista" (Briceño Ramírez, 2016: 95). En este marco, Marini, como militante del MIR y representante de la TMD, encuentran que la revolución socialista es el único camino para superar el subdesarrollo y la dependencia ${ }^{12}$. Esta postura se distancia de posiciones reformistas como las del PC, uno de los partidos con mayor peso dentro de la UP13, con quién Marini debate directamente en los estudios sobre Chile. Las críticas apuntaban tanto a la orientación tácticaestratégica sostenidas por el PC, como a la caracterización del modo de producción de América Latina en ese momento histórico, de la que se desprende dicha orientación táctica-estratégica.

\footnotetext{
${ }^{12} \mathrm{~A}$ pesar de que Marini y los intelectuales nucleados en torno a las TMD también debatieron con posicionamientos políticos estratégicos como los del foquismo, en los estudios sobre Chile, Marini se centró en los debates sostenidos con el PC.

${ }^{13}$ La UP se conformó en el año 1969 y se encontraba integrada por el Partido Socialista, el Partido Comunista, el Partido Radical, el Movimiento de Acción Popular Unitario (MAPU), la Acción Popular Independiente y el Partido Social Demócrata. En 1971 se incorporaron la Izquierda Cristiana, el MAPU Obrero Campesino y el Partido de Izquierda Radical. El MIR no formó parte de la alianza que conformaba la UP, si bien en un primer momento manifestó su apoyo al nuevo gobierno.
} 
Como punto de partida de su análisis, Marini destacó que, si bien no hay una diferencia de lectura política entre estos partidos respecto a la crisis del sistema de dominación de la burguesía, si se presentaron interpretaciones en disputa en relación a sus políticas de alianzas, los vínculos entre el partido y el movimiento de masas y la actitud a asumir frente al gobierno.

Respecto a las políticas de alianzas, Marini sostuvo que las diferencias entre el MIR y el PC, "no residían en el enemigo fundamental a combatir: la gran burguesía y el imperialismo, ni tampoco en la necesidad de establecer cierto grado de compromiso con las capas medias burguesas y pequeños burguesas" (Marini, 1976: 27), sino que ésta se encontraba en la determinación del bloque revolucionario. Como ya señalamos, la línea hegemónica del PC, en este momento histórico, fue una política de alianzas amplia, basada en una concepción etapista de los procesos revolucionarios, que llevó a privilegiar los vínculos con determinados sectores de la burguesía. Estrategia política que se sostuvo sobre una lectura teórica que consideraba que en América Latina regía un sistema feudal. Dicha interpretación fue considerada por los teóricos de las TMD como el principal error de la línea hegemónica del PC, que se basó en un deficiente análisis económico-social. La experiencia cubana puso en jaque la idea de "atraso" como nota distintiva de nuestras sociedades, a la vez que propuso un nuevo esquema interpretativo de las clases sociales, complejizando las visiones estancas en relación al problema de la existencia de una burguesía nacional y su papel en los procesos revolucionarios.

Las políticas de alianzas privilegiadas por el PC, lo condujeron, desde la perspectiva del MIR, a aceptar las reglas de juego establecidas por el sistema de dominación burgués, sin generar ningún tipo de cambio que transforme el sistema de raíz. Incluso, el tipo de alianza con la burguesía que propone el PC, en Chile, tal como muestra Marini, tuvo como contrapartida la articulación con sectores conservadores como la DC. En contra de esta línea estratégica, el MIR sostuvo que el bloque revolucionario debía tener como eje, al "proletariado organizado e incluir a las amplias masas proletarias y semi-proletarias de la ciudad, así como a las capas empobrecidas de la pequeña burguesía" (Marini, 1976: 27). Como sostiene Briceño Ramírez (2016: 95) la intelectualidad de la época reconoció al proletariado como la clase revolucionaria, posicionó a "la organización partidaria como vanguardia y estableció que dicha vanguardia debía tener una estructura político-militar, asumiendo la necesidad de implementar la lucha armada como estrategia bajo objetivos políticos y sustento teórico".

La prioridad política del MIR se centró en la organización de un movimiento de masas que se consolide como un poder alternativo al Estado burgués. En una coyuntura marcada por el agudizamiento de las contradicciones interburguesas y en el ascenso del movimiento popular, el MIR veía condiciones propicias para el desarrollo de una crisis revolucionaria que culminé en el derrocamiento de la burguesía y el imperialismo, así como, en el establecimiento de un Estado popular y revolucionario, que reemplace al Estado vigente. Por su parte, si bien el PC reconocía la importancia del desarrollo del movimiento de masas en el proceso revolucionario, su política subordinaba este proceso al gobierno. Para el PC, "toda acción de masas no autorizada y legitimada por el gobierno constituía, en última instancia, algo que afectaba a la estabilidad del mismo" (Marini, 1976: 29). A diferencia de esta postura, el MIR no lo entendía como un elemento desestabilizador del gobierno, sino que debía reforzarse y convertirse en órganos que sean tanto de combate popular como gérmenes de una nueva organización estatal (Marini, 1976: 29).

Para Marini, el hecho de que haya dos líneas en la conducción del movimiento de masas, impidió que se conforme un bloque unitario que confluya en un gobierno revolucionario. Además, la estrategia de alianzas y las políticas en el gobierno del PC impidieron la organización 
del movimiento de masas, al mismo tiempo que, fueron funcionales a la continuidad del sistema capitalista burgués.

\section{Desenlace de la lucha de clases}

Tal como señalamos, para Cueva (1974) el punto débil de las TMD es el análisis de las clases y su lucha, ya que parecería que en estos estudios los únicos protagonistas de la historia son las oligarquías y burguesías. A partir del análisis que realizamos en el presente trabajo, podemos observar cómo esta crítica puede ser discutida debido a que Marini en ningún sentido dejó de lado el rol del movimiento de masas urbano y rural para explicar el ascenso al gobierno de la UP, como su desarrollo. Sin embargo, consideramos que dicha crítica asume pertinencia en el momento en que el autor describe el desenlace de la lucha de clases. Al explicar el proceso contrarrevolucionario, que lleva a la caída del gobierno de Allende, Marini adjudica un papel central a la pequeña burguesía, dejando en un segundo plano del análisis la capacidad de reacción, influencia y presión del movimiento de masas.

El desenlace de la lucha de clases toma prioridad en el estudio de Marini en el momento que comienza a describir la gesta de la contrarrevolución burguesa de septiembre de 1973. Para explicar dicho proceso el autor retoma la dimensión económica, siendo el eje de análisis las políticas económicas de la UP. Como militante del MIR el autor presentó una disputa explícita con el PC, sector hegemónico del UP, con el cual, como ya vimos, tuvo diferencias respecto a la estrategia revolucionaria. En relación a las políticas económicas las críticas fueron dirigidas, en términos generales, a la falta de un intento por transformar de forma estructural la sociedad y economía burguesa, buscando soluciones dentro de las mismas lógicas del capital. En este sentido, las críticas se dirigieron a la negativa de la UP de transferir a los trabajadores el control real de la producción; así como a que no hubo una modificación de la estructura de consumo y producción, sino que la reactivación de la economía reprodujo las mismas dinámicas.

Marini destaca dos momentos en relación a cómo las políticas económicas de la UP se articularon con la acción política de las capas medias burguesas y pequeñas burguesas. Un primer momento, donde éstas lograron ser neutralizadas por el gobierno y, un segundo momento, en el cual pasaron a formar parte de la oposición. En los primeros años, según Marini, las políticas económicas de la UP se centraron en "desbloquear el desarrollo de las capas medias burguesas y pequeño burguesas, así como de atender a las exigencias de las masas populares en materia de salarios y consumos" (Marini, 1976: 35). Medidas como, la nacionalización de empresas monopólicas relacionadas con la producción de bienes corrientes, que aplastaron a las pequeñas y medianas empresas y, una fuerte redistribución del ingreso que impactó en la demanda de bienes de consumo, llevaron a que los sectores pequeños burgueses se inclinaran hacia el gobierno, dificultando a la gran burguesía nuclearlas bajo su dirección política. Sin embargo, la neutralización de la burguesía por el gobierno empezó a mostrar sus límites cuando el estrangulamiento del sector externo produjo el desabastecimiento de bienes tanto de consumo corriente, como de materias primas y bienes de capital. Esta situación desató el surgimiento y la expansión del "mercado negro", de la mano del acaparamiento y especulación de precios, comandado por el gran capital. Además de las ventajas económicas que esto proporcionaba a la gran burguesía, Marini destacó la centralidad de los beneficios políticos, ya que "ofrecía a las capas medias burguesas y pequeñoburguesas, la oportunidad de asociarse al negocio de la especulación" (Marini, 1976: 38) y así, volcarse a la esfera del gran capital. De esta forma, con un gobierno presionado por el desabastecimiento y las oportunidades abiertas por 
el gran capital, la pequeña burguesía encontró su lugar en la oposición. A su vez, el mercado negro y la especulación contrarrestó las medidas de redistribución de ingreso impulsadas por el gobierno, generando un enfrentamiento en el plano del consumo entre la pequeña burguesía asalariada y las capas pobres de la ciudad.

El rol político y económico que juega la pequeña burguesía es central para explicar las consecuencias políticas de la crisis económica. La principal de estas consecuencias fue para el autor, la agudización de la lucha de clases, que enfrentó a las clases fundamentales (burguesíaproletariado) a la vez que fortaleció un proceso de unificación y homogenización al interior de las mismas. Este enfrentamiento fue considerado por Marini como un momento único en la historia latinoamericana, donde se materializó el protagonismo y capacidad de lucha de las masas explotadas en el proceso revolucionario. En este contexto, Marini destacó la capacidad del movimiento obrero de organización, cohesión ideológica y de posicionamiento contra la política reaccionaria de la burguesía: "quien quiera que haya vivido el proceso chileno no puede menos que admirar la respuesta de los trabajadores ante la ofensiva desarrollada implacablemente por el capital en contra de sus condiciones de existencia" (Marini, 1973). Ni la capacidad del movimiento obrero de dar respuesta, ni los intentos del gobierno por enfrentar la contraofensiva burguesa, fueron suficientes para frenar el proceso contrarrevolucionario, que articulaba el gran capital, el militar-fascismo, las fuerzas imperiales en la región y la mediana y pequeña burguesía. Así, el golpe de estado de septiembre de 1973 clausuró la posibilidad de que triunfe la revolución socialista en Chile.

\section{Algunas consideraciones finales}

El recorrido por el análisis de los estudios sobre Chile de Marini, en conjunto con sus desarrollos teóricos contemporáneos, nos muestra que difícilmente pueda comprenderse los aportes del autor al margen del escenario político y social en el que el mismo se encuentra produciendo y debatiendo. Considerar el escenario de producción, así como la diversidad de obras realizadas por Marini, nos permite tener una mirada integral contrarrestando aquellas críticas que adjudican un carácter limitado a los análisis históricos y estudios teóricos del autor. En este sentido, recuperamos las críticas de Agustín Cueva (1974), quien sostiene que los teóricos de la dependencia, privilegian elementos económicos-estructurales en sus explicaciones y excluyen a las clases populares, cuestionando, así, la ausencia de articulación del análisis estructural con el de la lucha de clases en dichas teorías. En este trabajo buscamos mostrar cómo Marin articula elementos estructurales y superestructurales para comprender y analizar la experiencia chilena a fin de la década del 60 y comienzo de los años 70.

En términos generales, reconocemos que hay una preponderancia de la dimensión estructural en los diferentes análisis de Marini, sin embargo, esto no implica una visión sesgada de la realidad, más bien, se desprende de la misma perspectiva marxista. De hecho, el análisis de las condiciones estructurales es, en el materialismo histórico, un momento clave para el análisis de la realidad social, lo cual no supone que el papel asignado a esta dimensión sea el de determinación mecánica del resto de los aspectos constitutivos de aquella. Al mismo tiempo, en el momento de análisis estructural encontramos elementos políticos, ideológicos y culturales, que median y se filtran a las explicaciones estructurales que da el autor. Lo que reafirma la complejidad del análisis y la imposibilidad de distinguir taxativamente estas dimensiones. 
El ejercicio metodológico que guió nuestra investigación -un análisis que buscó articular la principal obra teórica del autor con sus estudios sobre la coyuntura chilena- nos permite mostrar la complejidad y el carácter dialéctico de los estudios y aportes teóricos de Marini. En primer lugar, para explicar el ascenso de la UP realizó un análisis económico estructural guiado por la teoría económico política del capitalismo dependiente, sin dejar de lado elementos políticos e ideológicos. Así, tuvo en cuenta como las políticas del gobierno de Frei Montalva impactaron en la disputa electoral de los sectores dominantes y, a su vez, incorporó al movimiento de masas como elemento central para comprender tanto el período de debilitamiento del gobierno de Frei Montalva y de ascenso de la UP.

En segundo lugar, identificamos que, en la caracterización de las clases sociales, Marini, utilizó categorías propias que se desprende de su teoría económica, como la de superexplotación pero su análisis no se limitó al lugar que ocupan las clases en la estructura productiva. De este modo, explicó el lugar de la pequeña burguesía y su rol político a partir de sus posiciones ideológicas; a la vez que, al considerar el accionar político de las clases trabajadoras y campesinas, las reconoció como actores políticos y no como meros instrumentos de dominio burgués. En este sentido, Marini lejos está de dejar fuera de su análisis el movimiento de masas como elemento central para comprender tanto el período de debilitamiento del gobierno de Frei Montalva y de ascenso de la UP, como el del gobierno de Allende. Si bien muchas de las conclusiones a las que arribó el autor pueden ser cuestionables, no puede afirmarse que dejó fuera el movimiento de masas como variable de análisis, tal como afirma Cueva. Sin embargo, reconocemos que, a la hora de abordar el desenlace de la lucha de clases, el actor principal, identificado por el autor, es la pequeña burguesía. Aquí es donde las críticas de Cueva se vuelven relevantes ya que para Marini es el papel que juega la pequeña burguesía, en última instancia, lo que desencadena la contrarrevolución. Parecería ser que el rol político de la pequeña burguesía es el único factor responsable de la caída del gobierno de Allende, descuidando la injerencia de otros elementos como, por ejemplo, el imperialismo estadounidense y la capacidad de organización del movimiento obrero y campesino.

En estos últimos tiempos nos encontramos con una multiplicidad de estudios teóricos y análisis coyunturales que sobredimensionan las variables políticas, sin poder tener una mirada clara sobre las transformaciones sociales, políticas y económicas ocurridas en la región. Es así que, recuperar y volver a ponderar elementos estructurales en los análisis coyunturales se vuelve una tarea urgente en el contexto actual. Los aportes de Marini se presentan como una puerta de entrada a estos desafíos ya que nos brindan herramientas para formular el problema de la articulación de la política y la economía en coordenadas que reconstruyan un vínculo orgánico entre la acción política, el Estado y los procesos de acumulación de capital. Elementos fundamentales para pensar los procesos sociales y políticos en la región, en tanto y en cuanto, la consideración de la especificidad latinoamericana se realiza a partir de una lectura atenta a la articulación dialéctica de escalas globales y locales.

\section{Bibliografía}

AMARAL, V. S. (2006) "A investida neoliberal na América Latina e as novas determinações da dependencia". En Disertación Maestría en Economía. Minas Gerais: Programa de Posgrado en Economía, Universidad Federal de Uberlândia. 
ASTARITA, R. (2010) Economía política de la dependencia y el subdesarrollo. Tipo de cambio y renta en la Argentina. Buenos Aires: Universidad Nacional de Quilmes.

BAMBIRRA, V. (1978) Teoría de la dependencia: una anticrítica. México: Era.

BEIGEL, F. (2006) "Vida, muerte y resurrección de las "teorías de la dependencia”. En Crítica y teoría en el pensamiento social latinoamericano. Buenos Aires: CLACSO. Recuperado de http://biblioteca.clacso.edu.ar.

BRICEÑO RAMÍREZ, L. (2016) "Vania Bambirra y la alternativa insurreccional a incios de los años 70", Revista Izquierda, núm. 28, Santiago de Chile, pp. 93-113.

CARDOSO, F. H. (1977) "La originalidad de la copia: La CEPAL y la idea de desarrollo", Revista de la CEPAL, núm. 4.

CEPAL (1951) Estudio económico de América Latina 1949. Nueva York: Naciones Unidas.

CUEVA, A. [1974] (2007) "Problemas y perspectivas de la teoría de la dependencia". En Entre la ira y la esperanza, y otros ensayos de crítica latinoamericana, Prometeo, Buenos Aires.

DOS SANTOS, T. (2002) Teoría de la dependencia. Balances y perspectivas. México: Plaza y Janés.

Luxemburg.

(2011) Marxismo y ciencias sociales: una revisión crítica. Buenos Aires: Ediciones

ENGELS, F. (1980) Cartas a José Bloch. Recuperado de https://www.marxists.org/espanol/me/cartas/e21-9-90.htm.

FURTADO, C. (1982) El desarrollo latinoamericano. El trimestre económico. México: Fondo de Cultura Económica.

KATZ, C. (2016) "El surgimiento de las teorías de la dependencia", Revista o olho da historia.

Recuperado de http://www.lahaine.org/katz/.

LÖWY, M. (2007) El Marxismo en América Latina: antología, desde 1909 hasta nuestros días. Chile: LOM.

LOZOYA, I. (2016) "Social scientists from the left-wing party and discussions about power in Chile (1970-1973)”, Universum, vol.31, núm.2.

MARINI, R. M. (1966) "La dialéctica del desarrollo capitalista de Brasil". En América Latina, Dependencia y Globalización. México, D.F.: Siglo XXI

[1973a] (2015) "La dialéctica de la dependencia". En América Latina, Dependencia y Globalización. Buenos Aires: CLACSO, Siglo del Hombre editores.

[1973b] (2015) "En torno a Dialéctica de la dependencia (postscriptum)". En América Latina, Dependencia y Globalización. Buenos Aires: CLACSO, Siglo del Hombre editores.

(1976) El reformismo y la contrarrevolución. Estudios sobre Chile. México: Era.

MARTINS, C.E. (2015) "Ruy Mauro: marco del pensamiento contemporáneo". En Ruy Mauro Marini, América Latina, Dependencia y Globalización (antología por Martins, C.E.) México: Siglo, XXI.

MARX, K. [1859] (2008) Prólogo a la contribución a la crítica a la economía política. México: Siglo XIX.

(2009a) El Capital, t.I.v.2.Buenos Aires: Siglo XXI.

(2009b) El Capital, t III.v.6. Buenos Aires: Siglo XXI.

PRESBICH, R. (1986) El desarrollo económico de la América Latina y algunos de sus principales problemas. Naciones Unidas, CEPAL.

RODRÍGUEZ, O. (1980) La Teoría del subdesarrollo de la CEPAL. México: Siglo XXI.

SIGAL, S. (1991) Intelectuales y poder en la década del sesenta. Bs.As.: Puntosur.

SVAMPA, M (2016) "La dependencia como eje organizador". En Debates latinoamericanos. Indianismo, desarrollo, dependencia, populismo. Buenos Aires: Edhasa.

TERÁN, O. (1993) Nuestros años sesentas. La formación de la nueva izquierda intelectual argentina. Bs. As.: Siglo XXI. 